\title{
Kritizistische oder empiristische Deutung der neuen Physik?
}

\author{
Bemerkangen zu Ernst Cassirers Buch „Zar Einsteinschen \\ Relativitätstheorie ${ }^{\mu}$.
}

Von 10 Trity Sehliek.

Ein unverwischbarer, unverä̀nßerlicher Charakterzug der kritischen Philosophie ist ihre Verwurzelung in der exakten Wissenschaft. Wie Kant selbst nach wohlbegründeter (besonders von Cohen verfochtener) Meinung mit seiner Erkeñntniskritik das Ziel einer philosophischen Rechtfertigung der Newtonschen Naturprinzipien verfolgte, só streben die neakąntischen Schalen danach, die Wahrheit der kritischen Grundgedanken dadurch zu beweisen, daB sie ihre Brauchbarkeit und Fruchtbarkeit auch für die Physik der neaen Zeit darzutun suchen. Es wurde dem Neukantianismus nieht schwer, mit der Entwicklang der Naturwissenschaft Schritt. zu halten, als sie vön der mechanischen zar energetischen und schließ̧lich zur elektrodynamischen Wèltansicht iuberging - ist aber seine Kraft and Flastizität auch groß genug, um den Sprung mitzumachen, durch den die Physik sich in unsern Tagen anf eine neae Bahn begab? ' Ich glaubte diese Frage verneinen za müssen za einer Zeit, als nur ganz wenige Versuche vorlagen, die Spezielle ReIativitätstheorie dem kritizistisehen Standpunkt zũ assimilieren, und als die Allgemeine Theorie überhaupt noch nicht abgeschlossen war. Es schien mir, daß die zu einer philosophischen Aufklärang and Rechtfertigung jener Theorie nötigen Prinzipien viel eher aus der empiristischen als aas der Kantschen Erkenntnistheorie entnommen werden können ${ }^{1}$ ); und auch bei späteren Gelegenheiten fand ich keine' Veranlassung, diesen Standpunkt auf-

1) Die philosophische Bedeutung des Relativitätsprinzips, Zeitschrift für Philosophie und phîlosophische Kritik, Bd. 159. 
zugeben, zumal die bald darauf glïcklich vollendete Allgemeine Theorie einem Gedanken zum Siege verhalf, der auf extrem empiristischem Boden (nämlich im Positivismús Machs) erwachsen war; Aber bei der Bedentung and Schwierigkeit der Frage ist "es Pflicht, die Sachlage bei jedem ernsten Anlaß erneát zn prüfen. Einen solchen Anlaß saltellt das Erscheinen des Buches von Ernst Cassirer ${ }^{1}$ ) dar, und so folge ich gern der Aufforderang der Schriftleitang der Kantstudien, dem Problem an der Hiand dieses Baches eine neue Untersuchang zu widmen, die freilich aus äußeren Gründen nur in ganz karzer Fassung gegeben werden kann.

Cassirer hat sich in seiner Schrift den Nachweis zam Ziel gesetzt, daß die philosophischen Grandlagen der Relativitätstheorie nur im Bereiche des Kritizismns gefunden werden können, genaner in derjenigen Form der kritischen Ansicht, die er gern als logischen Idealismus bezeichnet. Er stellt sich die Aufgabe, durch erkenntnistheoretische Analyse zu entscheiden, „ob die Theorie in ihrem Ursprung and ihrer Entwicklung als, Beleg and Zengnis für den kritischen oder als Zengnis für den sensualis tischen Erfahrungsbegriff zu gelten hat" (S. 26).

Angesichts dieser Formulierung müssen sich aber sogleich Bedenken erheben: Ist das Problem wirklich auf diese Alternátive zurückführbar? gilt hier ein tertium non datur? Sicherlich gibt es einen Empirismas, der vom Sensualismas verschieden ist and sich auf ihn nicht reduzieren läBt - das ist historisch wie sachlich leicht ersichtlich. Wenn also geżeigt wird (und das ist wohl nicht schwer), daß die Relativitätstheorie ans rein sensualistischen Prämissen nicht za verstehen ist, so wird hierdurch allein weder die Nótwendigkeit noch anch die Zalässigkeit der kritizistischen Interpretation der Theorie bewiesen, es sei denn, man faBte den Begriff des logischen Idealismus so weit, dab jene Alternative eben erlaubt wird. Dann aber schwebt or in Gefahr, seine entschiedene Färbang and damit seinen philosophischen Wert zu verlieren, die heterogensten Meinnngen wïrden sich in ihm vereinigen lassen. An einigen Stellen scheint Cassirer in der Tat za so allgemeinen Formulierangen za neigen, dab die Abgrenzung seines Kritizismus andentlich za werden droht. Wir mïssen den Grenzlinien nachzugehen suchen.

1) Ernst Cassirer, Zur Einsteinschen Relativitảtstheorie, Erkenntnistheoretische Betrachtungen, Berlin 1921. 
Um eine feste Grundlage für die folgenden Betrachtungen herzustellen, muß ich mit wenigen Worten sagen, welche anentbehrlichen Merkmale ich mir mit dem Begriff des Kritizismus verknüpft denke. Eine solche Festlegung ist durchaus nötig für jede Diskassion über die Verträglichkeit der Relativitätstheorie mit der kritischen Erkenntnislehre, denn nur auf diese Weise wird das störende Hineinspielen der Fragen der Kant-Interpretation vermieden; die Diskassion bleibt solange nnergiebig, als jeder sich des nicht ungewöhnlichen Arguments bedienen kann, der andere lege eben die Kantsche Meinung nicht richtig aus.

Folgendes also sei vorausgeschickt. Alle exakte Wissenschaft, deren philosophische Rechtfertigang unzweifelhaft das erste Ziel der von Kant begnündeten Erkenntnislehre bildet, beraht auf Beobachtungen und Messungen. Bloße Empfindungen and Wahrnehmungen sind aber noch nicht Beobachtungen und Messungen, sondern sie weiden es erst dadurch, daß sie geordnet und interpretiert werden. Die Bildung der physikalischen Gegenstandsbegriffe setzt also fraglos bestimmte Prinzipien der Ordnung tnd Interpretation vorans. Das Wesentliche des kritischen Gedankens sehe ich nun in der Behauptung, daß jêne konstitutivèn Prinzipien synthetische Urteile a priori seien, wobei zum Begriff des Apriơri das Merkmal der Apodiktizität (der allgemeinen, notwendigen, unümgänglichen Geltang) unabtrennbar gehört. - Ich bin zwar überzengt, mit dieser .Erklärung Kants eigene Meinung richtig zu treffen, aber selbsst w̧enn weder er noch seine Anhänger̈ diềser Art von Kritizizmas je gehaldigt hätten, bliebe ja die sachliche Richtigkeit oder 'Falschhëit der. folgenden Aufstellangen davon ganz unberïhrt, und auf diese allein kommt es bei einer Untersuchung an, die sich anf das Systematische, nicht auf das Historische richtet.

Die wichtigste Folgerung, aus der eben entwickelten Ansicht ist, dab' ein Denker, der die Unentbehrlichkeit konstitutiver Prinżipien zur wissenschaftlichen Erfahrung überhaupt einsieht, deswegen noch nicht als Kritizist bezeichnet werden darf. Ein Empirîst kann z. B. sehr wohl das Vorhandensein solcher Prinzipien anerkennen; er wird nari leugnen, daß sie synthetisch und a priori im oben bezeichneten Sinne sind.

Cassirer erkennt, daß "Empirismus und Idealismus sich in bestimmten Voránssetzungen begegnen. Beide gestehen hier der Erfahrung die entscheidende Rolle za - und beide lehren anderer- 
seits, daß jede exakte Messung allgemeine empirische Gesetze voraassetzt" (S. 94 f.). Aber indem er sich dann der drin̈genden Frage zuwendet, „wie wir za jenen Gesetzen, auf denen die Möglichkeit aller empirischen Messung beraht, gelangen und welche Art der Geltung ... wir ihnen zugestehen" (S. 95), stellt er dem Krițizismus nur die sensualistische Ansicht unter dem Namen des nstrengen" Positivismus gegenüber. Mit vollem Rècht verurteilt ex den von Mach gelegentlich unternommenen Versuch, selbst analytisch-mäthematische Gesetze gleich Dingeñ za behandeln, „deren Eigeñschaften man durčh unmittel̉bare Wahrnehmüng $a b_{-}$ lesen Kann" (S. 95) - jedoch damit ist nicht der logische Idealismus bewieșen, sondern nur der Sensualismus widerlegt. Zwischen beiden bleibt die empiristische Ansicht stehen, nach welcher jene konstitativen Prinzipien entweder Hypothesen oder Kon'ventionen sind; im ensten Falle sind sie nicht a priori (denn es mangelt ihnen die Apodiktizität), im zweiten sind sie nicht synthetisch. Wie steht 'es mit dem Nachweis, daß die Grundsätze der Einsteinschen Physik nicht diesen Charakter tragen, sondern als synthetische Sätze a priori anzusprechen seien?

Kant selbst rechnete, wie gar nicht zu bezweifeln ist, zu den gegenstandskonstituierenden synthetischen Prinzipien a prioxi die Axiome der euklidischen Geometrie und der Galileisehen Kinematik. Und die Mehrzahl der Kantianer hat auch nach der mathematischen Entdecknng der nichtenklidischen Geometrien an der enklidischen Naturauffassung als der einzig möglichen festgehalten, indem sie (sehr deutlich z. B. Riehl und Hönigswald) erklärten, der enklidischen Geometrie komme in der Tat die von Kant ihr zugeschriebene anschauliche Notwendigkeit za, während die andern Geometrien nur begriffliche Denkbarkeit besäßen, die ja der Kantschen Lehre nicht widerstreitet.

Nan ist die Spezielle Relativitätstheorie mit den Sätzen der Galileischen Kinematik, die Allgemeine anßerdem noch mit den Sätzen Euklids nnvereinbar. Wer die Einsteinsche Theorie annimmt, mab die Lehre Kants in ihrer arsprünglichen Form ablehnen; man muh, wie auch Cassirer mehrfach betont, einen Schritt über Kant hinaus tun. Aber darauf kommt es uns hier garnicht an. Der Kritizismus, wie er oben definiert warde, könnte dessenungeachtet der nenen Theorie gegeniber sich behaupten and bewähren, ja noch größere Triumphe feiern; daza wäre nur nötig, daB die letzten Grundlagen der Theorie sich eben als synthetische 
Sätze von schlechthin notwendiger Geltung für alle Erfahrung enthüllten. Welches sind diese Sätze?

Denn das ist wohl za beachten: wer die Behanptang des Kritizismus anfstellt, der muB, sollen wir ihm Glanben schenken, die Prinzipien a priori auch wirklich angeben, die den festen Grand aller exakten Wissenschaft bilden müssen. Für die Transzendentalphilosophie, sagt Cassirer mit Recht (S. 78), sind Raum und Zeit nicht Dinge, sondern "Erkenntnisquellen". Es mab also eine Angabe der Erkenntnisse gefordert werden, deren Quelle z. B. der Raum ist. Der kritische Idealist muß sie mit derselben Bestimmtheit und Dentlichkeit bezeichnen, mit der Kant anf die zn seiner Zeit einzig bekannte und anerkannte Geometrie and „allgemeine Bewegungslehre" hinweisen konnte. Alle die, welche die Relativitätstheorie vom Kantschen Standpunkt aus beurteilt baben, wiesen darauf hin, dab es sich in ihr, um die empirische (d.h. hier: durch physikalische Methoden gemessene Zeit) and um den empirischen Raum handelt, and sie stellen ihnen die Kantsche ${ }_{n}$ reine Anschanung" von Raum und Zeit gegenüber als dasjenige, was jene empirischen Konstruktionen erst möglich macht und folglich von jedem Fortschritt der Phýsik, der immer nar das Empirische betreffen kann, schlechthin anberührt bleiben maß. Durch diese Wendung wird die Problemlage nicht geändert, sondern nur andẹrs ausgedrückt, dénn die reine Anschanng ist eben die $\mathrm{Er}$ kenntnisquelle jener Grundsätze a priori;' deren man zar Konstruktion der empirischen Zeit und des empirischen Raums bedarf, fürr manche ist sie einfach ein zusammenfassender Terminus für den Inbegriff jener Grundsätze selbst; in jedem Falle kann die Existenz eines "reinen Ráumes" and einer „reinen Zeit" überhaupt nur dadurch erwīiesen werden, dạß man das System der dazugehörigen synthetisch-apriorischen Grundsätze tatsächlich anfzeigt oder wenigstens eine eindentige Anweisung gibt, wie es zu finden - ist. Es kann nicht genug , betont werden, daß ein Anhänger der kritischen Philosophie sich nür durch Vorweisung eines solchen Urteilssystems lègitimieren kann. Jeder Versuch, Eìnstein mit Kant zu versöhnen, muß in der Relativitätslehre synthetisch-apriorische Prinżipien aufdecken; sonst ist er von vornherein als gescheitert zu betrachten, weil er nicht einmal zu der richtigen Problemstellung vorgedrungen ist.

Cassirer sieht das Problem nätürlich in seiner richtigen Bedeutang, und an zwei Orten seines Buches scheint er eine nähere 
Bestimmang des Inhaltes dèr vom logischen Idealismus behaupteten reinen Anschauung zu geben. An der érsten Stelle (S. 84) erblickt er ihn in dem Begriff der' Koinzidenz der "Weltpunkte", auf welche die Allgemeine Relativitätstheorie bekanntlich alle Naturgesetze zurückführt. Aber ich glaube, daB gèrade diese "Koinzidenz" sich garnicht als bloßer Inbegriff and Knotenpunkt apriorischer Sätze auffassen läßț, sondern żunächśt durchaus Rèpräsentant eines psychologis chen Ërlebnisses des Zứsamenfallens ist, so wie etwa dàs Wort "gelb" ein einfáches nicht mehr definierbares Farberlebnis bezeichnet. Nur so vermag sie die von der Theorie ihr zugewiesene Vermittlerrolle zwischen Realițät und naturwissenschaftlich-begrifflicher Konstruktion zu spielen. Mit andern.Worten: wir haben eine empirische Anschaung vor uns ${ }^{1}$ ).

Eine zweite Antwort anf die Frage, was denn an synthetischapriorischen Sätzen über den Raum jetzt noch übrig bleibe, gibt Cassirer S. 101, wo er sagt: „Denn das, Apriori' des Raumes ... schlieBt . . . keine Behauptang über eine bestimmte einzelne Struktar des Raumes in sich, sondern geht nur auf jene Funktion der ,Räumlichkeit überhaupt', die sich schon in dem allgemeinen Begriff des linienelements als solchen - ganz abgesehen von seiner näheren Bestimmung - ausdrückte". Diese Formụlierang, die aussagen will, daß es überhanpt so etwas wie ein Linienelement in der Naturbeschreibung geben müsse, kann jedoch kaum befriedigen. Depn welcher Axiomenkomplex ist es, der in jener Behauptung beschlossen sein soll? Die Axiome der Stetigkeit können es nicht sein, denn die schon von Riemann ins Auge gefaBte Möglichkeit diskontinuierlicher Raumbestimmungen ist durch die móderne Quantentheorie in greifbare Nähe gerückt worden. Und welche andern Axiome man auch wählen möge : es ist nicht einzusehen, waram gerade sie die allein notwendige Raumstruktur konstitaieren sollen, da doch andere von nicht geringerer „Evidenz" dem Fortschritt der Physik zam Opfer fielen.

Hier erscheint jede inhaltliche Behauptung, so allgemein sie auch sein möge, schon zu speziell, und es ist durchaus konsequent, wenn man auf die Frage, welches denn nun die letzten synthetischen Grundsätze a priori aller Naturwissenschaft sind, die Ant-

1) Dies ist auch der eigentliche Sinn meiner Ausfuhrungen in „Raum und Zeit", 8. Auf. 1920, S. 83. 
wort erteilt (die ich einer freundlichen brieflichen Mitteilung Cassirers entnehme): ,eigentlich nar der Gedanke der ,Einheit der Natar' d. h. der Gesetzlichkeit der Erfahrung überhaupt, oder vielleicht kürzer der ,Eindeutigkeit der Zuordnung: “. Damit scheint mir aber die Gefahr unentfliehbar hereingebrochen zu sein, die ich oben als unvermeidliche Folge einer zu groben Umfangsweitang des kritischen Gedankens bezeichnete. Denn nun dürfte es nicht mehr möglich sein, jemals eine physikalische Theorie als Bestätigung der kritizistischen Philosophie' anzusprechen: diese müßte vielmehr mit jèder Theorie, sofern sie nur die Bedingungen der Wissenschaftlichkeit erfüllt, in gleicher Weise und ohne die Möglichkeit einer Selektion vereinbar sein. Einheitliche Naturgesetzlichkeit ist sicherlich die conditio sine qua non der Wissenschaft, weil, wie Cassirer selbst sagt (S. 45), „der allgemeine Gêdanke der Invarianz und Eindentigkeit ... in irgend einer Form in jeder Theorie der Natur wiederkehren muß". Auch für den Empiristen sind, wie Cassirer (S. 95) anerkennt, die Gesetze „das eigentlich Bleibende and Substantielle ${ }^{\star}$, auch der Empirist glaubt an die Einheit der Natur, an die Gesetżlichkeit aller Erfahrung, nur meint er, daß sich ihre Gültigkeit, ihre objektive Notwendigkeit durch keine transzendentale Deduktion oder sonstwie erweisen lasse. Hier kann sich der Kritizist auf keine physikalische Theorie berufen, denn jede beweist durch ihre Bewährung in der Erfahrung nur die tațächliche, nicht die notwendige Geltung des Satzes von der Einheit der Natur.

-Wie ein roter Faden zieht sich durch Cassirers Buch der mit den glänzenḋsten Mitteln überlegener philosophisch-historischer Kultur geführte Nachweis, daß die Relativitätstheorie dem in der Entwicklung der exakten Wissenșchaft von Platon bis heute immer richtungweisenden İdeal nicht , etwa widerspricht, sondern im Gegenteil seine zur, Zeit vollkommenste Erfüllung darstellt; daß die von ihr statuierte Relátivität der Maßbeștimmungen keineswegs einen Verzicht auf streng eindentige objektive GesetzmäBigkeit bedeutet, sondern, im Gegenteil der Weg ist, 'zu allgemeinsten Geșetzen zu gelangen and letzte Invarianten aufzudecken. Ein neuerer Aufsatz Cassirers (im Dezemberheft der Neuen Rundschan) ist im wesentlichen dem gleichen Nachweis gewidmet. So notwendig und vérdienstlich es war, durch solche Ausfülarungen ñaheliegenden laienhaften Mißverständnissen der Einsteinschen Theorie entgegenzatreten and sie in den gebührenden Abstand 
von jedem sophistischen "Relativismas" skeptischer Färbang za rücken, so wird damit doch nur bestätigt, daß die Relativitätslehre, weil sie eben eine wissenschaftliche Theorie ist, natürlich eine Aufstellung, nicht eine Anfhebung allgemeinster, objektiv gültiger Gesetze bedentet.' Das Einsteinsche Weltbila läßnt die Einheit der Natar vollkommener hervortreten als das Newtonsche; aber nicht, weil es dem kritischen Gedanken gệnäBer wäre, sondern weil es, schon am physikalischen Erkenntnisbegriff gemessen and noch unabhängig von der letzten philosophischen Interpretation, eine höhere Erkenntnisstufe darstellt.

Die Frage, ob dem yon Cassirer so tief durchdachten logischen Idealismus der Nachweis der Richtigkeit der Behauptung gelungen sei, daß nur auf dem Boden der kritizistischen Eirkenntnislehre die Relativitätstheorie sich philosophisch begründen und rechtfertigen lasse - diese Frage vermögen wir nach dem Vorangehenden gerade in bezug auf den entischeidendeñ Punkt nicht $z$ L bejahen: die Lehre von den synthetischen Urteillen a priori als den konstruktiven Prinzipien der exakten Naturwissenschaft erfährt durch die neue Theorie keine nnzweidentige Bestätigung. Cassirers Darlegungen scheinen mir keine überzengende Anweisung zu geben, wie die Wunde geheilt werden kann, die der ursprüñnglichen Kantschen Ansicht durch den Umstarz der Euklidisehen Physik geschlagen ist. Aber damit ist noch nicht gesagt, daß das Verhälțis zwischen Transzendentalphilosophie und Relativitätstheorie non überhaupt als ein rein negatives erwiesen wäre; an andern Punkten könnten bedeatsame Berührungen beider Gedankenkreise stattfinden, wichtige Gemeinsamkeiten sich offenbaren.

Es liegt überaus nahe, in der kritischen Lehre von der' Idealität des Ranmes nnd der Zeit eine enge-natïrliche Verwandtschaft mit den Gedanken der Relativitätstheorie zu suchen. Man hat in der Tat die Wesenlosigkeit, die den Raum" der Einsteinschen Naturlehre vor dem starren Raum Newtons (und ebenso die Zeit) anszazeichnen scheint, als eine willkommene Bestätignng der Kantschen Philosophie betrachtet. Auch Cassirer vertritt diese Auffassung. Im AnschluB an meine Bemerkung, daih nach der allgemeinen Relativitätstheorie nur einer unauföslichen Einheit von Raum, Zeit and Stoff noch das Prädikat der Wirklichkeit zakomme (Raum und Zeit S. 67), meint ex, diese Einsicbt gehöre ${ }^{2 n}$ den Grandlehren des kritischen Idealismus selbst“ (S. 93); und ferner: „die ideelle Trennung des reinen Raumes and der reinen 
Zeit von den Dingen (genauer von den empirischen Erscheinungen) duldet nicht nur, sondern fordert geradeza ihre empirische ,Union" " (S. 94). Dies letztere ist freilich richtig, denn Raum und Zeit sind als Formen der Anschauung von dem in ihnen geformten Stoff ebenso wenig trennbar, wie umgekehrt der Stoff ohne eine Form sein kann. Aber die von der Relativitätstheorie behauptete ,Union', die ich darch jene Bemerkung zu treffen suchte, ist eine viel innigere als die Einheit von Stoff und Form, über welche die Transzendentalphilosophie nirgends hinausgegangen ist. Wenn daher Cassirer fortfährt: "Diese Union hat die allgemeine Relativitätstheorie in einem neuen Sinne bewährt und erwiesen ...", so ist der Ton durchaus anf das Wort neu zu legen. Dieses Neue.wird gänzlich verkannt von E. Sellien ${ }^{1}$ ), welcher sagt:

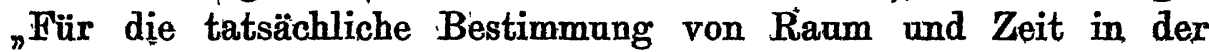
Erfahrung 'gehören Raum, Zeit und. Körpèr zusammen. Dieser Satz ist keine Errungenschaft der Einsteinschen Theorie, wie Schlick mit so viel Emphase behauptet, er ist längst bekannt, und widerlegt Kants Lehre vón der, rein en Zeit durchaus nicht, weil er sie garnicht berührt": Es ist jedoch ein schlechthin fundamentales Miß̣verständnis: der Allgemeinen Relativitätstheorie, wenn man glaubt, meine oben erwähnte Bemerkung so auffassen zu dürfen, als solle in ihr "nür negạtiv die Sonderexistenz von Zẹit und Raum gégenüber der Materie (und umgekehrt) gelengnet wèrden $\rightarrow$ das wäre . freilich eine längst bekannte Trivialität. Sondern die gegenseitĭge Ábhängigkeit von Ratmo, 'Zeit und Materie geht-in der Einsteinschen Theorie viel tiefer; nach ihr ist es z: B. unmöglich, von den Abmessungen einer Raumgestalt ohne Rücksicht auf die Art ihrer materiellèn Erfüllúng za sprechen. Daß die Raumlehre in dièser Weișe zum Zweige der Physik wird; verdient allerdings mit großer Emphase hervorgehoben zu werden. Nur Riemann hat diesen Gedanken mit völliger Klarheit vơrwíggenommen: dem Kritizismus lag er nicht bloß fern ${ }^{2}$ ),

1) Die erkenntnistheoretische Bedeutung der Relativitätstheorịe, Kieler Dissertation, 1919, S. 37; anch als Ergänzungsheft 48 der "Kạntstudien" erschienen.

2) Man hat zwar auch in diesem Punkte Kant zum Vórląufer Einsteins erklären wollen. 'Auf. Grund ein̈iger Remerkungen in Kants erster Schrift, „Gedankén vọn der wahren Schätzung der lebendigẹn, Kräfte" sagt Ilse Schneider (Das Raum-Zeitproblem bei Kant und Einstein, Berlin 1921, S. 70): "Kant weist alsọ als erster auf den Zusammenhang von Geometrie und Physik, speziell Gravitation, hiñ". Aber Kants Fersuch, die Dreidiménsionalitát des Raumes mit der Formel des Newtọschen Gravitationsgesetzes in Bezzehung zu bringen, bedeutet 
sondern er scheint ihm zu' widersprechen, weil er es unmöglich macht, Raum und Zeit als bloße Formen in dem bisherigen Sinne aufzufassen, deren Gesetze von ihrem Inhalt unabhängig za behandeln sind. Wenn Einstein von der Durchführung der allgemeinen Relativität ,bemerkt hat, sie nehme dem Raum und der Zeit "den letzten Rest physikalischer Gegenständlichkeit", so glaubt Cassirer, „daß die Theorie hierin nur dem Standpankt des kritischen Idealismus die bestimmteste Anwendung and Durchführong innerhalb der empirischen Wissenschafft selbst verschafft" (S. 79). Legen wir aber - wie ès Cassirer (S. 13) mit Recht als erste Aufgabe des Erkenntnistheoretikers fordert - den Sińn des Terminus "physikalische Gegenständlichkeit" restlos klar, so stoßen" wir wieder anf das eben geschilderte Ergebnis, dem die Lehre von der Idealität von Zeit und Raum nur nach seiner negativen Seite hin gerecht zu werden vermag: es zeigt sich nämlich, ${ }^{*} \mathrm{da} \beta$ mit der „Gegenständlichkeit" dem Raum und der Zeit zwar jede irgendwie beschaffene Unabhängigkeit von der Materie abgesprochen wird, daß aber der Rest, der dann voì physikalissch Räumlichen und Zeitlichen übrig bleibt, im Verein mit der Materie sich auch derselben Realität erfrent wie dieșe. Einstein selbst hat gelegentlich ausgesprochen, daß der physikalische Raum auch nach der Allgemeinen Relativitätstheorie Realität habe, nur keine selbständige. Das Räumliche und Zeitliche erhalten also einen Sinn, in dem sie nicht mehr blob als ,Formen' in der gewohnten Bedentung angesehen werden dürfen, sondern sie gehören jetzt za den physikalischen Bestimmungsstücken der Körper; die ,Metrik' bedeutet nicht etwa blob eine mathematisehe Messung des physikalisch Realen, sondern drückt selbst dessen Vorhandensein aus. Raum und Materie treten eben, wie Cassirer es durchaus treffend ausdrückt, „nicht mehr als verschiedene Klassen physikaliseher Objektbegriffe anf" (S. 61). Wenn man also der Meinung ist, die Einsteinsche Physik weise „in dieser Hinsicht weniger Widersprüche zar kantischen transzendentalen Ästhetik anf, als. irgend eine frühere Physik ${ }^{\alpha}$ ), so scheint mir darin eine Verkennung der

nichts weniger als eine Vorahnung der "Vereinigung von Geometrie und Physik im erkenntnistheoretischen Sinne, hat vielmehr gar nichts damit zu tun. Mit silnlichem Rechte könnte man hier auf die Cartesianische Identifizierung von Substanz und Ausdehnung hinweisen, die auch Cassirer erwahnt (S. 60), obne daB er aber ihre wahre Bedeutung ibbertriebe.

1) Ise Schneider, 1. c. S. 65. 
positiven Seite der Einsteinschen Raum- and Zeitlehre zn liegen. Es wäre auch verwunderlich, wenn die Kantsche Erkenntnistheorie in so deutlichem. Widerspruch stehen sollte $\mathrm{zar}$ Newtonschen Natarlehre, deren philosophische Rechtfertigung eines ihrer vornehmsten Ziele war. -

Mag aber auch die rechte Würdigung der allgemeinsten Relativität (Kovarianz gegenüber beliebigen Substitutionen in der Sprache der Theorie) von kritizistischen Prinzipien aus schlecht gelingen: vielleicht könnten sie doch insofern einen tragfähigen Unterbau Einsteinscher Lehren liefern, als sie wemigstens zu dem Grundsatz der Relativität aller Bewegungen (Kovarianz gegenüber einer bestimmten Gruppe von Substitationen) in einem günștigen Verhältnis stehen. Natürlich ist von einem philosophischen System nicht zu verlangen, da "es diesen Grundsatz als Theorie durchführe, wohl aber kann er sich aus ihm als, unentbehrliches Postulat ergeben. Ișt auch dies noch zu viel gefordert, so darf man zum allermindesten erwarten, daß. jener. Grundsatz, nachdem er von anderer Seite einmal anfgestellt war, sofort als kongenial erkannt und von dem Sysstem mit größter Energie angeeignet werde. Tàtsächlich wäre der Kritizismus hierz̈u aus seinen Prämișsen heraus sehr-wohl imstande gewesen; dennoch hat er in seinen historischèn Erscheinungsformen von den eben aufgestellten Forderungen keine , erfüllt. Es war vielmehr der Positivist Mach, der das allgemeine Relatìvitätsprinzip zuerst mit Nachdruck zu einem Postalat der Naturbeschreibung erhob." Er verlangte - and zwar wirklich ans philosophischen Gründen - eine solche Formulierung der Naturgesetze, daß z. B. die Rotation der Eirde gegen die Fixsterne mit gleichemo Recht als 'eine entgegengesetzte Drehung des Stèrnhimmels am die Erde aufgefaßt werden könnțe. Um Kants Stellung zu diesem Gedanken kennen zu lernen - der ja zu seiner Zeit genau so möglich war - lese man die Metaphysișchen Anfangsgründe der Naturwissenschaften, wo er im I. Hauptstäck in dẹr Anmerkang zum Grundsatz I, und im IV. Hauptstück im Lehrsatz 2 und "der Allgemeinen Anmerkang zur •Phänomenologie das Problem bespricht: Er fühlt dort (wie Leibniz, Huyghens und andere) dürchaus das Bedürfnis, die Relativität alle r Bewegung aufrecht.zu enhalten. Während aber Newton erkannte, dab dies mit seiner Mechanik unvèreinbar sei und für sie folgerichtig (vermutlich nicht ganz leichten Herzens) die absolute Bewegung postulierte, sucht Kánt dadurch nach einem Ausweg, da 
er neben den Gegensatz der relativen ùnd absolnten denjenigen der "wahren" und ${ }_{n}$ scheinbaren" Bewegung setzte! ${ }^{1}$.

Cassirer hat bereits in "Snbstanzbegriff und Funktionsbegriff" (1910) die Frage der Relativität der Rotation behandelt' (S. 230 ff.). Es ist höchst bemerkenswert, mit welchem Scharfsinn er schon damals die Konsequenzen der Machschen Auffassung überblickte. Er sagt nämlich (1. c. 246): „Die positivistischen Bedenken gegen den ,reinen ${ }^{7}$ Raum and die ,reine Zeit beweisen daher nichts, weil sie zu viel beweisen würden: sie müßten, konsequient zu Ende gedacht, anch jede Darstellung physsisch gegebener Körper in einem geometrischen System, in welchem es feste Iagen und Entfernungen gibt, verwehren". Hier and in den der zitierten Stelle vorhergehenden Entwicklungen werden also vom kritizistischen Gesichtspunkte aus im wesentlichen gerade die Konsequenzen verworfen, zu denen sich die Naturwisșenschaft jetzt gezwtungen sieht.

GexwiB hat Cassirer Recht mit seiner Meinung, daß die Bestätigang der Machschen Relativitätsbehauptung für sich noch keinen zwingenden Beweis für die Notwendigkeit einer empiristischen Interpretation der Einsteinschen Theorie liefere (S. 97) aber ein höchst bedeutsames Indizium bleibt sie doch. Urid zwar, wie ich glaube, kein trügerisches. Denn das erkenntnistheoretische Motiv, das Mach und Einstein (sei es mit Recht oder Unrecht) zu dem Postulat der Relativität aller Bewegungen führte, war der Satz, daB Unterschiede des Wirklichen nur dort angenommen werden dürfen, wo Unterschiede im. prinzipiell Erfahrbaren vorliegen. Diese fandamentale Regel ist öfters ausgesprochen worden, auch von Metaphysikern wie Leibniz, bei dem sie gleich in zwei Gestalten erscheint, nämlich als principiam identitatis indiscernibilinm and als principe de l'observabilité (in letzterer Form führt es auch Cassirer S. 37 an); aber von der Anfstellung bis zum konsequenten Festhalten und Durchführen der Regel ist noch ein großer Schritt. Wird der Grandsatz jedoch in seiner wahrhaft fundamentalen Bedeutung erkannt und gewürdigt, so läßt er sich, wie ich glaube, zum obersten Prinzip aller empiristischen Philosophie erheben, zar

1) Ise Schneider ( c. S. 14) zitiert die entsprechende Kantstelle beifällig, weil sie Kant als Gegner der absoluten Bewegung erscheinen 1ăBt, aber sie vergißt, daB jene Unterscheidungen gerade vom Standpunkt der Relativitätstheorie eine Cngeheuerlichleit darstellen. 
letzten Richtschnar, die bei der Stellungnahme zu jeder Einzelfrage maßgebend sein muß, und deren unerbittliche Anwendung auf alle Spezialprobleme ein Verfahren von höchster Fruchtbarkeit darstellt. Ist diese Auffassung richtig, so wäre damit allerdings der Zusammenhang der Relativitätslehre mit der empiristischen Erkenntnistheorie als ein innerlicher, streng sachlicher, als nicht bloß äußerer und zufälliger erkannt.

Im letzten Kapitel seines Buches betont Cassirer mit Nachdrack, dab der Raum und die Zeit der Relativitätstheorie eben Raum und Zeit der $\mathrm{P}$ hysik sind, nicht der Wirklichkeit schlechthin, sodaß ihnen etwa der Raum und die Zeit der Psychologie als etwas gänzlich Heterogenes gegenüberstehen. Es ist in der Tat von größter Wichtigkeịt, sich stets darüber klar za sein, daß man von Raum und Zeit in völlig verschiedenen Bedeutungen reden kann - am wiohtigsten gerade auch für den, dem es, schließlich auf die Erkenntnis des Zusammenhanges dieser verschiedenen Bedeutangen ankommt. Wènn ich an andern Stellen ${ }^{1}$ ) den psychologischen Raum (und die Zeit) als das rein Anschauliche dem physikalischen als einer rein begrifflichen Konstruktion gegenüberstellte, so war ich "mir wohl bewußt, daß die "Anschanng" bei Kant in einer ganz andern. Weise abgegrenzt wird. In diesem Punkte bin ich von einer Reihe von Kritikern mißverstanden worden. Cassirer erklärt ${ }^{2}$ ) Kants reine Anschaung als eine bestimmte "Methode der Objektivierung": das ist sie freilich a uch, aber ihr Wesen erschöpft sich nicht darin. Gewiß, wollte Kant alles Psychologische aus, ihr entfernen - aber ich werde mich niemäls überzengen können, dạß , es ihm gelungen ist: Denn es kann eben nicht gelingen ${ }^{3}$ ) ohne die Anwendung der einzigen Methode, die das rein Begriffliche der Geometrie vom Psychologisch-Anschaulichen zu trennen ermöglicht; das ist die Methode der impliziten Definition, die erst in der modernen Mathematik ansgebildet warde ${ }^{4}$ ). Ohne sie ist es nicht einmal möglich, die

- Idee eines reinen Begriff's za fassen und in seiner Ablösung von allen psychologischen Momenten $\mathbf{z u}$, verstehen. Kants reiner An- schauungsraum enthält also notwendig solche Moment,e, sie geben dem Raumbegriff den Inhalt, ohne den er für Kant nleer" wäre.

1) ${ }_{n}$ Raum und Żeit" 3, S. 81, "Allgemeine Erkenntnislehre", S. 301 ,

2). S. 123, 124, Anmerkung.

3) Wiè sich z B. aus den Ausfülurungen Selliens (1. c. S. 40) erkennen läBt.

4) Vgl. "Allgemeine Erkenntnislehre ${ }^{*}$, S. $30 \mathrm{ff}$. 
Kritizistische oder empiristische Deutung der neûen Physik?

Sein Raum ist ihm zwar identisch mit dem Raum. Newtons' (dies ișt auch die Meinung Cassirers, die ich stets geteilt habe; Sellien ${ }^{1}$ ) jedoch, den Cassirer sonst zastimmend zitiert, scheint ihr za widersprechen), aber der Newtonsche Raüm ist anch bei ihm ebeñ ein anschaulicher, noch nicht gereinigt von den Elementen, die wir noch als psychologisch bezeichnen müssen. Só ist Kants reine Anschaung - wie es auch durchaus der verbreiteten. Meinung von Raum and Zeit entspricht - ein Mittelding zwischen rein Begrifflichem und psychologisch Anschavlíchem; und da ich es fürr eins der wichtigsten Ergebnisse dèr modernen Theorie der exakten Wissenschaft halte (in diesem Punkte hat sich Henri Poincaré besonders große philosophische Verdienste erworben), daß es solch eine Mischung, solch ein Mittelding eben nieht gibt, so maßte ich einerseits die Existenz einer reinen Anschanung im Sinne Kants lenginen (Allgem. Erkenntnislehre, S. 302) und durfte von einer Vermengung des physischen Raumbegriffs, mit seinen sinnlichen Repräsentanten sprechen (Raum and Zeit, S. 83); andererseits mubte ich erklären, daß in der Lehre von den, blob subjek-" tiven Anschanungsformen eben insofern ein richtiger Kern zu finden ist, als sie noch von psychologischen Momentến nicht ganz entblöBt. sind. Diese Ansichten vermag ich àlso nicht aufzugeben.

Die Verfolgang des Bedeutungswandels der Termini Raum und Zeit durch die verschiedenen Gebiete des Geisteslebens gibt Cassirer Gelegenheit, seine Betrachtung der Relativitätslehre großzügig in umfassendere Zusammenhänge einzuordnen und außer dem Lichte der speziellen Erkenntniskritik anch die Strahlen der systematischen Philosophie auf sie za richten. So schliebt das Buch mit einem Umblick, dessen Weite der Höhe des eingenommenen Standpunktes entspricht. Wir scheiden mit dem Eindruck, dạ dieser Standpunkt über die Region des eigentlichen Kritizismus, doch schon hinausliegt, and dab es Cassirer nar hiexdurch gelang, der Relativitätstheorie philosophisch in dem Maße gerecht zu werden, wie es in' dem geistvollen and gedankenreichen Buche geschieht.

Gern willfahre ich der Aafforderung der Leitang der Kantstudien, bei dieser Gelegenheit noch über zwei andere Bücher zar Einsteinschen Lehre kurz za berichten, denn es handelt sich um

1) 1. c. S. 16. Dort ist von der Zeit die Rede; rom Ranm aber gelten die Argamente in gleicher Weise. 
Schriften, von denen za sprechen sich lohnt. Die erste, verfaBt von Max Born ${ }^{1}$, gibt eine glänzende, ausführliche Darstellang der Einsteinschen Lehre vom Standpunkt des Physikers aus. Sie fülllt in überaus trefflicher Weise eine sehr fühlbare Lücke der Einstein-Literatur, denn während die bis dahin vorhandenen gemeinverständlichen Einführungen in die Theorie bei der Besprechung ihrer physikalischen Grundbegriffe sich auf das notwendigste beschränkten, erscheint die Theorie in dem Bornschen Buche zum ersten Mal nicht vop ihrem natürlichen Hintergrunde abgelöst; sondern es wird gerade auf ihre Einordnung in das System der Physik großes Gewicht gelegt, klar treten die Zazuammenhänge' hervor, aus denen sie in Wirklichkeit erwachsen ist. Für den Nichtphysiker ist es von höchstem Werte, in diese Zusammenhänge eingeweiht zu werden, denn durch sie führt der naturgemäße Weg zum Verständnis. Born ebnet diesen.Weg nicht nur durch Vermeidung aller höheren Mathematik, sondern selbst Logarithmen and trigonometrische Funktionen kommen nicht vor. Die Hauptsache aber ist: das Buch ist durch und durch das Werk eines' philosophischen KKopfes. Das zeigt sich nicht etwa darin, daß Born den Gang seiner Darstellung durch philosophische Dentangen und Abșchweifungen unterbräche, sondẹn in der Höhe der Gesichtspunkte; die den Aufbau bestimmen, und in der tiefen Besinnung, die aùs der Behandlung des. Gegenstandes überall hervorleachtet. Es zeigt sich ferner vor allem in der kurzen philosophischen Einleitung, die geradezú klassisch anmutet in der Wärme tund der Prägnanz, mit der sie dẹn Grundgedanken vorträgt: daß das Absolute nur im Umkreis des Subjektiven zu finden ist, und daß der denkende Geist in die Sphäre der objektiven Geltung nur vordringen kann, indem er das Absolute opfert, um Erkenntnis des Relativen dafür einzutauschen. Fürwahr eine fundamentale Einsicht, die nicht nur in der theoretischen. Wissenschaft offenbàr wird, sondern nach meiner Überzeugung sich anch in der praktischen Philosophie bewährt.

Die zweite Schrift. ìst das Büchlein „Relätivitätstheorie und Erkenntnis a priori" von Hans Reichenbach (Berlin 1920). Es stellt zweifellos einen großen Fortschritt in der logischen Deutang der Einsteinschen Lehre dar.' Reichenbach leuchtet durch, eine

1) Die Relativitätstheorie Einsteins und ihre physikalischen Grundlagen gemeinverständlich dargestellt, mit 129 Abbildungen und einem Portrát. Sp̈ringer, Berlin 1920. 
Art axiomatischer Methode in sehr scharfsinnigen und sêlbștändigen Ausführangen in die logischen Grandlagen der Relativitätstheorie hinein und liefert dabei durch Aufdeckung gewisser versteckterer Prinzipien (er spricht 'z. B. von einem „Prinzip des approximierbaren Ideals", einem "Prinzip der normalen Induktion" usw.) einen wertvollen Beitrag zur Logik der exalkten Wissenschaft überhaupt. Er gelangt zu dem Resultat, daß Einsteins Theorie mit der ursprïnglichen Kàntschen Lehré nicht vereínbar sei, und er nimmt eine solche Umbildung des Aprioribegriffs vor, $\mathrm{da} B$ die Relativitätstheorie ihm nicht mêhr widerspricht tnd, wie er meint, der wichtigste Grandgedąnke der Kantschèn Philosophíe aufrecht erhalten bleibt. Diesen Grandgedanken glaubt er nämlich 'in der Einsicht zi finden, daß jede Erkenntriis nur durch die logische Voraussetzung gewisser Prinzipien möglich wird, die ihren Gegenstand tuberhaupt erst konstituieren. Solche Prinzipien nénnt er a priori, läßt aber das Merkmal der Apodiktizität fallen; sie sind also-nicht notwendig, and fortschreitende Erfahrung kann Anlab $2 n$ ihrer Modifikation geben. „Apriori bedentet: vor der Erkenntnis, aber nicht: für alle Zeit, und nicht: unabhängig von der Erfahrung" (S. 100): Nach dem oben (S. 98) Gesagten scheint mir der Boden des Kritizismus damit vollständig verlassen zu sein; and Reichenbachs Prinzipien a priori würde ich als Konventionen im Sinne Poincarés bezeichnen. Die Terminologie des Verfassers kann ich also nicht gutheißen, aber sachlich stimme ich in den meisten wesentlichen Ergebnissen durchans mit ihm überein. Selbst in den Fragen, in bezag anf welche or in der Schrift gegen mich Stellung nimmt, besteht in Wahrheit keine tiefgehende Verschiedenheit der Meinungen, wie eine briefliche Erläuterung beider Standpunkte nachträglich ergeben hat. Abèr anch für den, der diesen Standpankten fern steht, ist das Büchlein wertvoll, denn eine philosophische Leistang, die sich durch Originalität, Klarheit and Schärfe der Gedankenführung so auszeichnet wie die vorliegende, maB dem Leser auch dann Genuß and Vorteil bieten, wenn sie ihn zum Widerspruch anregt. 